\title{
Review
}

\section{The effect of noradrenergic drugs on the recovery of walking after spinal cord injury}

\author{
$\mathrm{H}_{\text {Barbeau }}{ }^{* 1,2}$ and KE Norman ${ }^{3}$ \\ ${ }^{1}$ School of Physical and Occupational Therapy, McGill University Montreal, Quebec, Canada H3G 1 Y5; ${ }^{2}$ Jewish \\ Rehabilitation Centre 3205 Place Alton, Goldbloom, Chomedey, Laval, Quebec, Canada H7V 1R2; ${ }^{3}$ School of \\ Rehabilitation Therapy, Queens University, Faculty of Health Sciences, Kingston, Ontario, Canada, K7L 3 N6
}

Clonidine, a noradrenergic agonist has been associated with improved walking in both spinal cat and spinal cord injured (SCI) subjects.

Objectives: The objective of this brief review is to compare the effects of clonidine on walking capabilities in SCI subjects with functionally complete and incomplete spinal cord injuries.

Study design/methods: Both oral administration and intrathecal injection of clonidine were investigated. A motorized treadmill was used and harness support provided in most of the SCI subjects as no walking capabilities could be observed overground. A single subject design was used in these chronic SCI subjects.

Setting: Canada and France.

Results: In complete SCI subjects while receiving clonidine, none of the subjects was able to initiate independent stepping. In contrast, the greatest effects were found in SCI subjects with injuries that are incomplete but still severely disabling while minimal effects could be observed in the more functional SCI subjects. These effects on walking are observed in measures of walking speed, and electromyographic and kinematic patterns. Regardless of effects on walking, however, a consistent decrease of the flexor reflex amplitude could be observed in all SCI subjects independent of the severity of the lesion.

Conclusion: This review demonstrated that clonidine could be a powerful anti-spasmodic drug in addition to improving locomotion in a limited number of SCI subjects. The mechanism, significance and implications of these results will be discussed.

Sponsorship: Fonds de la recherche en Santé du Québec, Echange Franco-Québec and network center of excellence in Neurosciences.

Spinal Cord (2003) 41, $137-143$. doi:10.1038/sj.sc.3101374

Keywords: spinal cord injuries; walking; reflexes; clonidine

\section{Introduction}

Spinal cord injury has an estimated incidence that varies greatly across developed countries. The range is from 2.1 to 123.6 per million of population. ${ }^{1-6}$ Recent epidemiologic studies revealed that the spinal cord injured (SCI) individuals are increasingly younger, arrive sooner at the hospital, and have less severe cord injuries. $^{7}$ These recent findings suggest that recovery of behaviour such as walking is becoming a possibility for an increasing proportion of this population. It is thus increasingly important to study the recovery of walking of SCI subjects who have an incomplete sensory and/or motor function loss. In the past decade, our laboratory

*Correspondence: H Barbeau, School of Physical and Occupational Therapy, Faculty of Medicine, McGill University, 3630 Sir-WilliamOsler Street, Montreal, Quebec, Canada, QC H3G 2Y5 has been investigating new therapeutic approaches to enhance the recovery of walking in SCI subjects. ${ }^{8}$

Following complete spinal cord transection in mammals, all of the descending systems degenerate and their neurotransmitters progressively disappear in their monoaminergic terminals. Noradrenergic agonists such as clonidine that act on receptors caudal to the transection have been shown both to initiate and to modulate the locomotor patterns as well to modify spinal reflexes. The present brief review will focus on the effect of noradrenergic agonists that mimic noradrenaline.

The $\alpha_{2}$ noradrenergic agonist, clonidine, has been used to induce locomotion in acute spinal cats. ${ }^{9-11}$ When clonidine or tizanidine was given during the first few days after spinalization, a well-coordinated 
locomotor pattern and full weight support of the hindquarters were progressively observed. In contrast, injection of dopamine or serotonergic agonist could not initiate locomotion during the same period. ${ }^{10}$

If no drugs are given, chronic spinal cats may achieve a stable locomotor pattern within a month if they are appropriately trained, ${ }^{12}$ and this finding has permitted the exploration of several pharmacological interventions in the modulation of an established locomotor pattern. The administration of clonidine or tizanidine led to increases in the maximal walking speed, the step cycle duration for the same treadmill speed, the excursions of the hip, knee and ankle, and the duration of the EMG bursts. In addition, the threshold current needed to elicit a small flexion reflex was two to three times higher after clonidine administration. These effects could be partially reversed by yohimbine, a noradrenergic antagonist, confirming that clonidine exerts a modulatory effect on the locomotor pattern through noradrenergic receptors. ${ }^{11}$ When clonidine was administered to chronic spinal cats that had developed a poor locomotor pattern, similar positive results were obtained. ${ }^{12}$

Rossignol's recent review $^{13}$ also reported that an injection of intrathecal noradrenaline but not clonidine in a cat, after a severe lesion of the ventral and ventrolateral funiculi, improved the locomotor pattern as well as doubled maximal walking speed, suggesting that noradrenaline does not seem to interfere with voluntary control of the animal, which is an important goal when introducing locomotor pharmacotherapy in SCI subjects.

On the basis of these animal findings, we believe that a rationale for locomotor pharmacotherapy can be developed for SCI subjects.

\section{The effect of clonidine in functionally complete SCI subjects}

In a placebo-controlled study, the effects of clonidine on the initiation of walking pattern were investigated in chronic SCI subjects with clinically complete spinal cord injury. They were supported and assisted in stepping by a body weight support (BWS) system and by at least two physiotherapists.

While receiving placebo, none of the subjects with complete SCI was able to initiate independent stepping. Instead, each subject was assisted in passive stepping on the treadmill while completely supported by the harness of the BWS system. The presence of regular flexor bursts is evident in Figure 1A, during assisted locomotion in the placebo session of a subject with complete SCI. Right and left medial hamstrings $(\mathrm{MH})$ showed a consistent alternating pattern (Figure 1B). The timing of this burst was coincident with the stretch applied to the hamstrings in mid and late swing, when the knee was passively extended as the leg was drawn forward by the physiotherapists in preparation for foot-floor contact. Passive flexion and extension of the knee, with the same subject seated, likewise consistently evoked a $\mathrm{MH}$ burst following administration of the placebo. During the stance phase a burst in the extensor muscles such as vastus lateralis (VL) and gastrocnemius (GA) could be

A

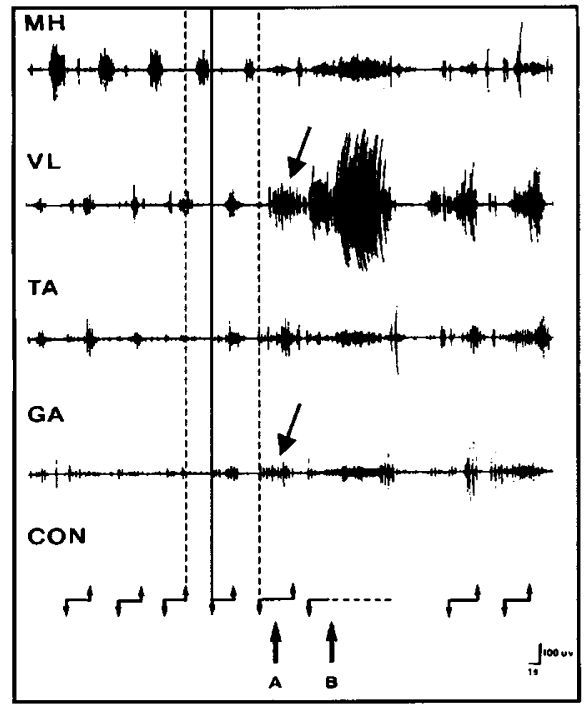

B

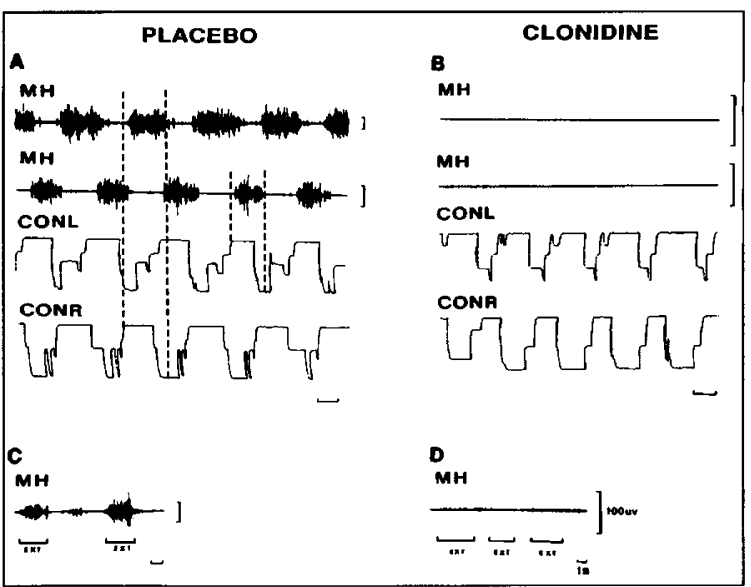

Figure 1 (A) EMG activity in right muscles for a complete SCI subject during assisted treadmill locomotion. Downward arrows indicate foot floor contact, upward arrows toe off, and horizontal lines reflect stance phase. The single arrow indicates when verbal instructions were given for the subject to attempt independent stepping with the right foot. The prolonged swing phase in the subsequent step reflects the dragging of his foot as he was unable to step forward successfully. The oblique arrows indicate the increased activity during the loading phase of the step cycle. (B) Bilateral medial hamstring EMG activity and footswitch traces of another complete SCI subject during assisted treadmill locomotion are presented for the placebo (A) and clonidine (B) sessions. Recordings of left medial hamstring EMG during passive knee extension for the placebo $(\mathbf{C})$ and clonidine (D) sessions are also presented. The duration of the passive extension was kept constant across the sessions. The vertical calibration indicated $100 \mathrm{UV}$ and horizontal calibration indicates $1 \mathrm{~s}$ 
observed as a result of the loading on the lower extremities. The amplitude of those extensors varied with the amount of weight on the lower extremities (see arrows in Figure 1A). However, during the clonidine session, both stretch reactions were abolished (Figure 1B). Furthermore, during assisted locomotion while the subject was receiving clonidine, the therapists reported a reduction in the resistance previously felt when pulling the leg forward. This reduction in stretch reactions in $\mathrm{MH}$ during locomotion was demonstrated in four out of six subjects with complete SCI while receiving clonidine. ${ }^{14}$

The absence of the locomotor pattern in subjects with complete SCI could possibly be explained by the chronic state, since they had not experienced locomotion for many years. It is also possible that the spinal central pattern generator for locomotion is more difficult to express in primates ${ }^{15}$ and depends more heavily on the presence of supraspinal influences. It is additionally possible that some forms of lower flexor afferent stimuli are required to trigger spinal locomotion, as demonstrated in spinal cats. It can be argued that a higher dosage of clonidine may be necessary to facilitate the expression of the locomotor pattern; however, the presence of adverse side effects is an important limiting factor. An alternative method of drug administration such as an intrathecal injection should also be investigated. ${ }^{16}$

These results have recently been replicated by Dietz et $a l^{17}$ who found that no stepping movements were evoked in five complete SCI subjects who received clonidine.

\section{The effect of clonidine in functionally incomplete SCI subjects}

In contrast, the most severely impaired among functionally incomplete SCI subjects showed a marked improvement in walking ability, as revealed by EMG and kinematic patterns.

The chronic SCI subjects still required manual assistance for most stepping with maximal BWS at minimal speed of the treadmill $\left(0.04 \mathrm{~m} \mathrm{~s}^{-1}\right)$. The temporal data from these assisted cycles are shown in Figure 2A, immediately above the raw EMG signals from two of these cycles. There was irregular activity of soleus throughout the cycle, with clonus accompanied by coactivation of tibialis anterior. The clonus was visible in the soleus by its characteristic $5-7 \mathrm{~Hz}$ bursting pattern. The vastus lateralis records show
A

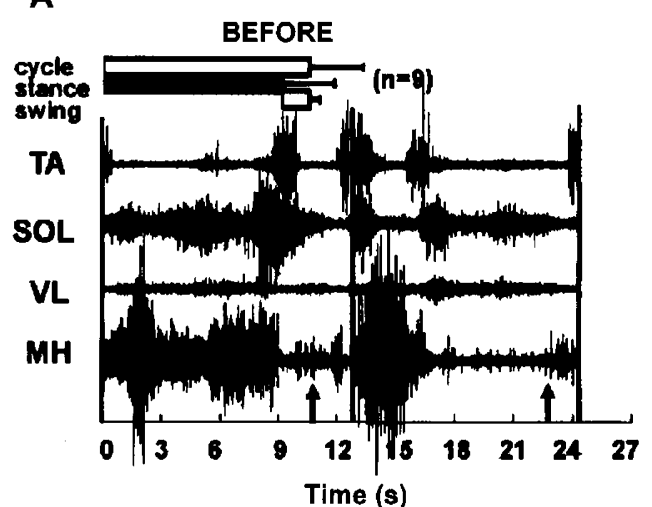

B

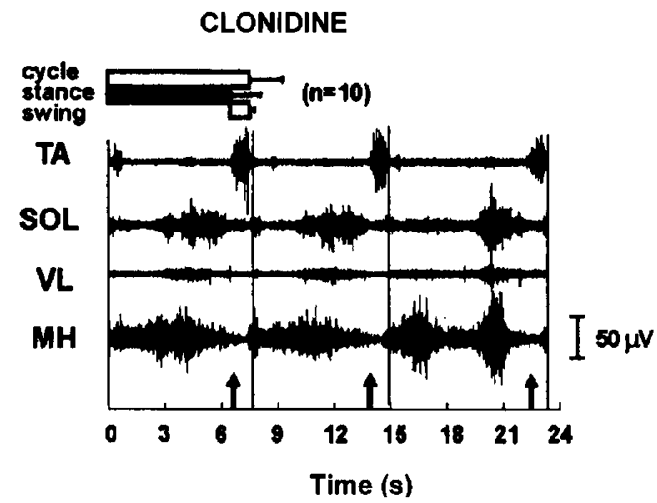

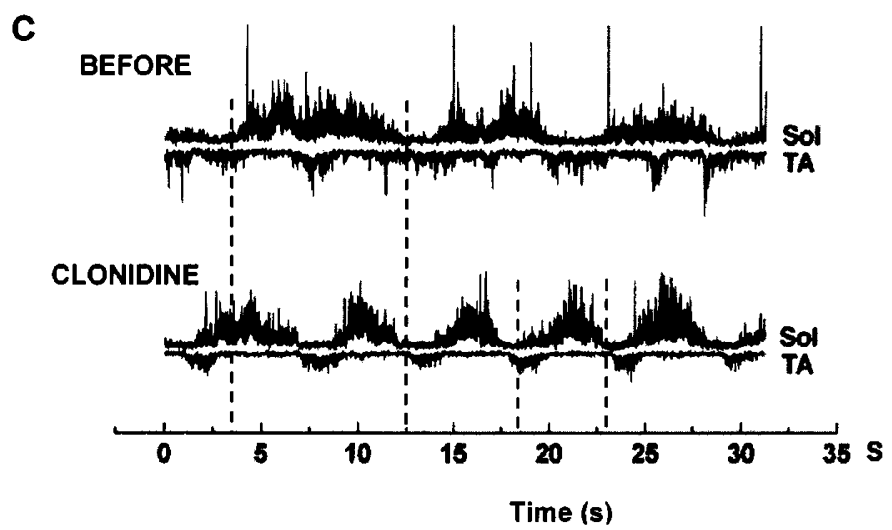

Figure 2 Raw EMG traces of several cycles from incomplete SCI subjects: (A) before (B) following oral clonidine administration $(0.25 \mathrm{mg})$ and $(\mathbf{C})$ before and following $60 \mu \mathrm{g}$ intrathecal clonidine administration. EMG traces are plotted as a function of time in seconds, vertical lines denote cycle boundaries and upward arrows along axes denote stance-swing transition within each cycle. TA, Tibialis anterior; SOL, soleus; VL, vastus lateralis; $\mathrm{MH}$, medial hamstrings. Vertical dotted lines indicate the cycle duration (modify from references 16 and 19) 
little cyclical modulation in activity. The medial hamstrings record shows some cyclical modulation with activity primarily during single limb support, in contrast to the usual pattern of activity for the hamstrings in which it is active mostly in double limb support prior to single limb support on the ipsilateral side. $^{18}$

A marked contrast of locomotion pattern with a regular pattern of muscle activity could be observed when the subject was receiving clonidine (Figure 2B). The soleus (SOL) and tibialis anterior (TA) show a much more reciprocal pattern than previously seen. The SOL activity usually begins in early stance and diminishes in late stance at which point the TA activity begins for its sharpest peak during the cycle. In addition, there is both a disappearance of clonus in soleus and a reduction in the prolonged high amplitude EMG activity in the medial hamstrings. ${ }^{14,16,19,20}$ This SCI subject changed from no walking to an increased walking speed up of $0.25 \mathrm{~m} \mathrm{~s}^{-1}$ (Figure 3A). A more normal activation pattern of the TA and SOL muscles has also been reported by other groups ${ }^{21-23}$ in case reports with clonidine and with tizanidine.

Recently, a brief report was published on the acute effects of an intrathecal injection of clonidine on the overground walking pattern of SCI subjects. ${ }^{16}$ Within a $30 \mathrm{~min}$ of clonidine injection, three of the
10 SCI subjects increased their maximal overground walking speed. The speed gains were maintained or increased over successive evaluations during the subsequent $6 \mathrm{~h}$.

As shown in Figure 2C, the TA showed a more regular activation pattern in the three subjects after intrathecal clonidine injection, and the co-contractions that had been regularly observed before injection of clonidine completely disappeared after $60 \mu \mathrm{g}$ clonidine injection (Figure 2C). The cycle duration also decreased from 9 to $4 \mathrm{~s}$ in this subject (see vertical bars in Figure 2C) related to an increase of walking speed (Figure 3A right side).

In the more functional SCI subjects, minimal effect of oral or intrathecal clonidine, regardless of dosage, could be observed on walking speed (Figure 3B) but a decrease in the flexor reflex and the Ashworth spasticity scale score was consistent in all the SCI subjects, whereas the H-reflex amplitude remained unchanged (not illustrated; see Remy-Neris et al. ${ }^{16}$ ) In the same subjects, the changes in walking speed, flexor reflexes and spasticity score were minimal on days when placebo injections were given. The reduction of signs of spasticity concurrent with the increase of walking speed suggest that intrathecal clonidine may provide a useful treatment for SCI subjects. These results are consistent with findings of changes in locomotor pattern and reduced excitability of spinal
A

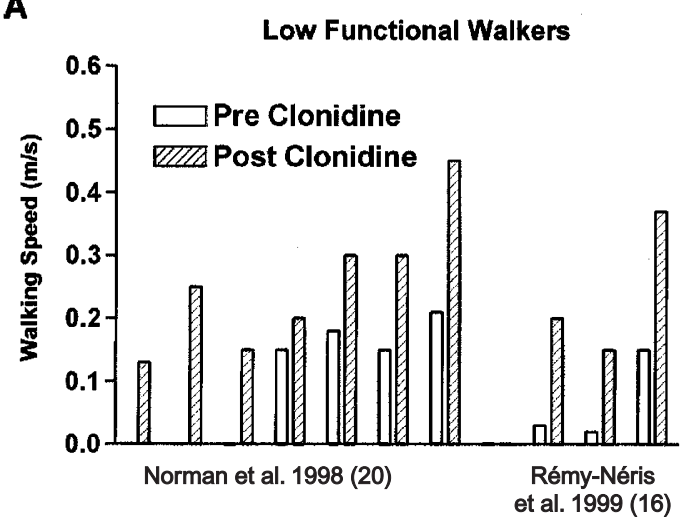

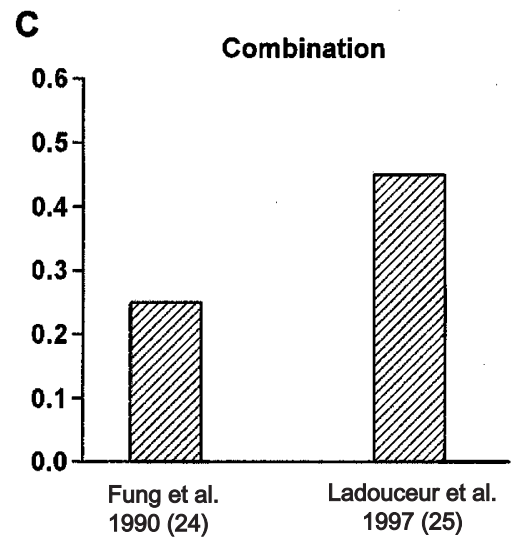

Functional Walkers

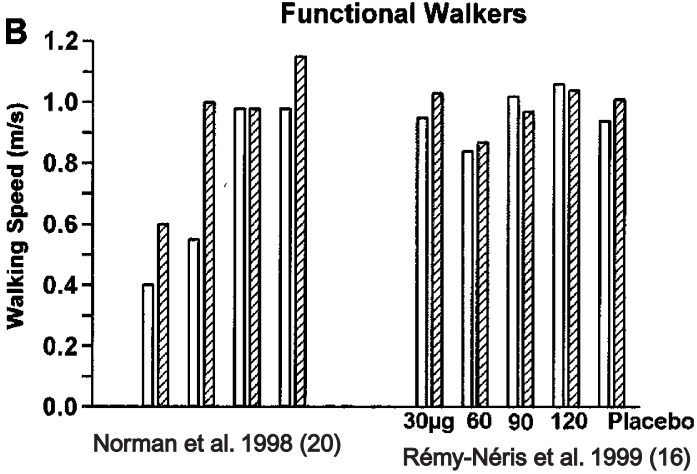

Figure 3 The effect of clonidine on walking speed in low SCI functional walkers (A) and in functional SCI walkers (B). (C) represents the combination of clonidine with locomotor training on walking speed 
reflexes with clonidine in chronic spinal cats ${ }^{11}$ but the relationship between change in reflex and change in locomotor behaviour needs further investigation.

\section{The combined effect of clonidine with locomotor training}

Figure 4 shows an example of the combined effects of cyproheptadine (a serotonergic antagonist) and clonidine (a noradrenergic agonist), together with an interactive locomotor training program in a chronic SCI subject that sustained a traumatic spinal cord injury due to a motor vehicle accident. He had no isolated voluntary movements in either lower limb. Limited by severe spasticity, this subject could stand with support but was unable to take one single step independently overground like the other SCI subjects reported in this brief review.

During the placebo evaluation, this spastic SCI subject required maximum body weight support $(50 \%$ BWS) and manual assistance to initiate stepping on the treadmill. His gait pattern was characterized by a flexed posture and a marked flexion in the hip and knee indicating the total incapacity to bear weight on the lower extremity even at the minimal treadmill speed. Furthermore, this subject frequently encountered flexor spasms as soon as the foot was lifted off the treadmill, giving rise to an excessive flexion in the hip and knee during swing (not illustrated). It can be seen from Figure $4 \mathrm{~A}-\mathrm{D}$ that the EMG profiles in this subject during the placebo evaluation were characterized by abnormal EMG timing. The MH and TA both showed a prolonged tonic burst throughout the gait cycle. GA showed similar tonic activity with clonus present during both stance and swing. Minimal activity was present in VL, possibly due to the incapacity to cope with weight acceptance during stance. Moreover, there was a marked variation of the stance-swing transition and prolonged swing duration due to prolonged flexor activity, as indicated by the solid line across the cycles in Figure 4A-D.

Following the administration of clonidine $(0.20 \mathrm{mg} /$ day) with cyproheptadine $\left(16 \mathrm{mg} \mathrm{day}^{-1}\right)$, in combination with locomotor training, the SCI subject gained the ability to walk unassisted and independently at full weight bearing on the treadmill at the speed of $0.26 \mathrm{~m} \mathrm{~s}^{-1}$. With the improved ability to cope with weight, there was a marked decrease in flexion at both the hip and knee joints during stance. The excessive flexion of both joints in swing was also markedly diminished. Trunk and lower limb abnormalities such as trunk leaning forward, lack of hip extension, knee sag, and short stride length were observed (not illustrated $\left.{ }^{24}\right)$. With locomotor training using BWS (Figure $4 \mathrm{E}-\mathrm{H}$ ), the SCI subject gained the ability to walk full weight bearing, and an EMG activation of VL muscle now occurred throughout the single limb support period of stance. The abnormal burst of $\mathrm{MH}$ activity became silent and was replaced by a burst in early stance. Another important change was the more
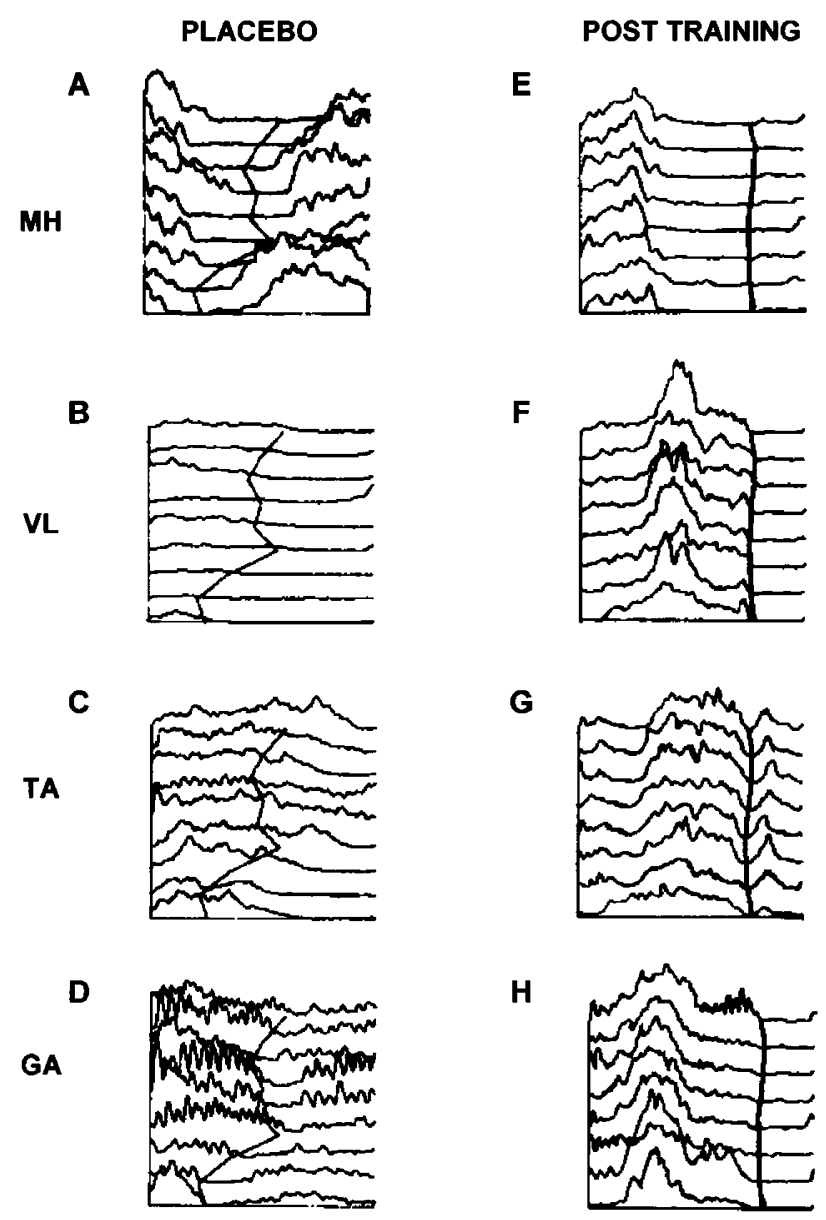

Figure 4 The combined effects of pharmacological intervention and interactive locomotor training on EMG profiles of an incomplete SCI subject during treadmill walking. EMG activity from $\mathrm{MH}, \mathrm{VL}, \mathrm{TA}$ and GA were normalized across 6-10 consecutive gait cycles, during the placebo $(\mathbf{A}-\mathbf{D})$ and post-training $(\mathbf{E}-\mathbf{H})$ evaluations. Solid line across the cycles depicts stance-swing transition

appropriate timing of GA, now limited to the stance phase with a marked reduction of the clonus. This resulted in a more regular stance-swing transition as indicated by the solid line across the cycle in Figure $4 \mathrm{E}-\mathrm{H}$ and a better kinematic pattern. ${ }^{24}$

Functionally, this chronic SCI subject could walk independently overground with a walker following medication and with elbow crutches following the combination with locomotor training. This was remarkable since this subject was wheelchair-bound at the beginning of the study. Moreover, the SCI subject showed a marked improvement in his endurance, from being able to take only a few assisted steps with maximal BWS and manual assistance during the placebo evaluation, to a much longer period, 5-8 min continuously and independently at full weight bearing following medication and locomotor training. ${ }^{26}$

Clonidine does not, however, appear to improve walking in all subjects; it is unclear why only three 
PHARMACOLOGY OF NOREPINEPHIINE

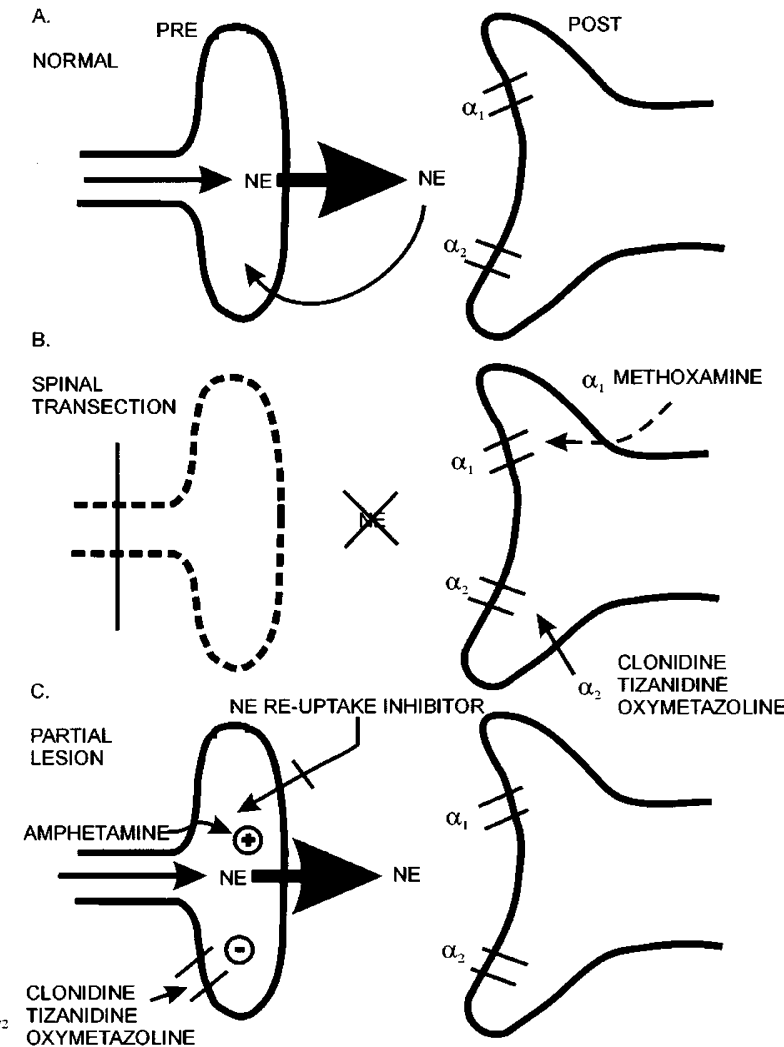

Figure 5 Schematic of the pharmacology of norepinephrine during intact situation (A), following complete spinal transection of the cord (B) and following a partial lesion of the cord (C) (see text for explanation)

among 11 incomplete SCI subjects improved in walking speed in the report by Remy Neris et al. ${ }^{16} \mathrm{It}$ was suggested that this might be due to the possible action of clonidine on presynaptic receptors, some of which are still present after an incomplete spinal cord lesion. Clonidine could then inhibit the release of the norepinephrine from the presynaptic terminals (Figure 5). To explore the possible mechanism related to this controversy, amphetamine, which releases norepinephrine from the presynaptic terminals, has been used in both animals and in humans. ${ }^{26}$ Amphetamine, which increases the brain and spinal cord levels of norepinephrine, has been shown to promote recovery of function in animal models of brain injury. The effectiveness in humans is still unknown but we know that low doses of amphetamine can be used without serious side effects in stroke subjects ${ }^{27}$ and need to be investigated in SCI subjects. Rossignol et al ${ }^{13}$ recently showed that norepinephrine but not clonidine enhanced locomotor activity in incomplete spinal cats. This could be due to actions of clonidine in the spinal cord. There are only noradrenergic postsynaptic receptors left after complete spinal section, and their activation by clonidine would enhance locomotor activity. When the section is not complete, clonidine acts primarily at presynaptic receptors, which reduce the release of NE to the spinal cord and so reduce locomotor activity. Further investigation is required to understand the effects on locomotion of various types of noradrenergic drugs to the spinal cord following both complete and incomplete injuries.

\section{Conclusion}

The present findings have general potential clinical implications. Clonidine could be a powerful antispastic drug in addition to improving locomotion in a limited number of SCI subjects. Future studies on the effect of clonidine on locomotion should explore a more complete dose-response curve and its action in combination with other drugs (e.g. serotonergic) and/ or in combination with other locomotor training strategies such as functional electrical stimulation. ${ }^{27}$ Finally, it will be important to identify features of SCI subjects that may predict who will benefit from such therapy.

\section{Acknowledgements}

Many thanks are due to Vira Rose for her contribution for secretarial support, Gevorg Chilingaryan for the figures and the statistical analysis. The Fonds de la Recherche en Santé de Québec (FRSQ) and the JRH foundation for their continuing support. From ASIA/IMSOP Symposium, Vancouver May 2002.

\section{References}

1 Botterell EH et al. A model for the future care of acute spinal cord injuries. Can J Neurol Sci 1975; 2: $361-380$.

2 Gjone R, Nordlse L. Incidence of traumatic paraplegia and tetraplegia in Norway: a statistical survey of the years 1974 and 1975. Paraplegia 1978; 16: 88-93.

3 Kraus JF. Injury of the head and spinal cord: the epidemiological relevance of the medical literature published from 1960 to 1978. J Neurosurg 1980; 53 Suppl: S3-S10.

4 Kurtzke JR. Epidemiology of spinal cord injury. Exp Neurol 1975; 48: $163-236$.

5 Minaire P et al. Epidemiology of spinal cord injury in the Rhones-Alps region, France, 1970-1975. Paraplegia 1978; 16: $76-87$

6 Shingu H, Ikata T, Katoh S, Akatsu T. Spinal injuries in Japan: a nationwide epidemiological survey in 1990. Paraplegia 1994; 32: 3-8.

7 Tator $\mathrm{CH}$ et al. Changes in epidemiology of acute spinal cord injury from 1947 to 1981. Surg Neurol 1993; 40: $207-215$.

8 Barbeau H, Rossignol S. Enhancement of locomotor recovery following spinal cord injury. Curr Opin Neurol 1994; 7: $517-524$.

9 Forssberg H, Grillner S. The locomotion of the acute spinal cat injected with clonidine i.v. Brain Res 1973; 50: $184-186$. 
10 Barbeau H, Rossignol S. Initiation and modulation of the locomotor pattern in the adult chronic spinal cat by noradrenergic serotonergic and dopaminergic drugs. Brain Res 1991; 546: 250-260.

11 Barbeau H, Julien C, Rossignol S. The effects of clonidine and yohimbine on locomotion and cutaneous reflexes in the adult chronic spinal cat. Brain Res 1987; 437: 83-96.

12 Barbeau H, Rossignol S. Recovery of locomotion after chronic spinalization in the adult cat. Brain Res 1987; 412: $84-95$.

13 Rossignol S, Chau C, Barbeau H. Pharmacology of locomotion in chronic spinal cats. In: Taylor A, Gladden MH, Durbaba R (eds). Alpha and gamma motor systems. New York: Plenum Press, 1996, pp 449-455.

14 Stewart JE, Barbeau H, Cauthier S. Modulation of locomotor patterns and spasticity with clonidine in spinal cord injured patients. Can J Neurol Sci 1991; 18: 321 332.

15 Fedirchuk, B et al. Pharmacologically evoked fictive motor patterns in the acutely spinalized marmoset monkey (Callithrix jacchus). Exp Brain Res 1998; 122: $351-361$.

16 Remy-Neris $\mathrm{O}$ et al. Effects of intrathecal clonidine injection on spinal reflexes and human locomotion in incomplete paraplegic subjects. Exp Brain Res 1999; 129: $433-440$.

17 Dietz V, Colombo G, Jensen L, Baumgartner L. Locomotor capacity of spinal cord in paraplegic patients. Ann Neurol 1995; 37: 574-582.

18 Winter DA. Biomechanical motor patterns in normal walking. J Motor Behav 1983; 15: $302-330$.

19 Norman KE, Barbeau H. Comparison of cyproheptadine, clonidine and baclofen on the modulation of gait pattern in subjects with spinal cord injury. In: Thilmann AR, Burke DJ, Rymer WZ (eds) Spasticity: Mechanisms and Management. Berlin: Springer-Verlag, 1993, pp $410-$ 425 .
20 Norman K, Pepin A, Barbeau H. Effects of drugs on walking after spinal cord injury Spinal Cord 1998; 36(10): $699-715$

21 Bastings E, Rapisarda G, Pepin JL, Delwaide PJ. Nouvelles approches pharmacologiques du traitement des troubles de la marche consecutifs à une lesion spinale. Rev Med Liège 1995; 50: 204-208.

22 Delwaide PJ, Pennisi G. Tizanidine and electrophysiologic analysis of spinal control mechanisms in humans with spasticity. Neurology 1994; 44 (suppl 9): S21-S27.

23 Knutsson E. Analysis of gait and isokinetic movements for evaluation of antispastic drugs or physical therapies. In: Desmedt JE (ed). Motor control mechanisms in health and disease. Advances in Neurology 39. New York: Raven Press, 1983, pp 1013-1034.

24 Fung J, Stewart JE, Barbeau H. The combined effects of clonidine and cyproheptadine with interactive training on the modulation of locomotion in spinal cord injured subjects. J Neurol Sci 1990; 100: $85-93$.

25 Ladouceur M, Pepin A, Norman KE, Barbeau H. Recovery of walking after spinal cord injury. Adv Neurol 1997; 72: $249-255$.

26 Gladstone DJ, Black SE. Enhancing recovery after stroke with noradrenergic pharmacotherapy: a new frontier? Can J Neurol Sci 2000; 27: $97-105$.

27 Unwin H, Walker-Batson D. No side effects after lowdose amphetamine administration in stroke rehabilitation. Stroke 2000; 31: $1788-1789$. 